\title{
"Autonomy" Replacing "Heteronomy": Transfer of Management Model of Urban Community
}

\author{
Jie Ma \\ Department of Law and Politics, Changzhou University \\ Changzhou 213164, China \\ E-mail: maleikejie@sina.com
}

\begin{abstract}
"Management model" is an important theoretical and practical proposition in study on urban community. Urban community is defined as a harmonious community with psychological and regional congruence. Rebuilding of a harmonious urban community is, as a matter of fact, a process of urban management model transferring from "heteronomy" to "autonomy". Urban community autonomy means self-governance of self-organization on community affairs. Transfer of urban community management model is a systematic project with reformation significance. On one hand, generation of the autonomy model is not a simultaneous behavior, but is dependent on creation and accumulation of system, law, economy and organization, etc. On the other hand, selection of appropriate reform means is based on consideration of transfer conditions and development stages.
\end{abstract}

Keywords: Urban community, Management model, Transfer conditions, Reform means

\section{Urban community: dispute and definition}

The bone of contention on urban community can be summarized into two aspects: one based on "psychological" level and the other based on "regional" level. The former stipulates qualitatively whether urban community exists and has strong "Toennies sentiment" and the latter stipulates quantitatively dispute on positioning of urban community and reflects strong practice orientation.

As the founder of the concept of "community", Toennies held a pessimistic attitude towards urban community. The sort of "community" he described is generally a sort of intimate interpersonal relationship in which everyone trusts and helps each other and is obedient to the authority. Community is an organism full of vigor, while society is mechanical aggregation and artifact. Traditional rural area is representative of community, while the newly emerging city is representative of the society. Community and society and rural and urban area are diametrically opposed, and transfer from "community" to "society" is just as "one steps to a certain strange place." The psychological community concept of Toennies provided the earliest root for the dispute of whether there exists urban community. Thus, the two view points of "theory of disappearance" and "theory of existence" were formed in the academic circle. According to the former view point, urbanization is power that damages traditional community and it makes features of "community" fade away and traditional community go to break up, community no longer existing in cities. By contrast, according to the latter, urbanization is not the necessary factor for community life to go to break up, and on the contrary, the sense of belonging in communities of areas with high degree of urbanization is also high, and urban community is not extinct, but changing and is becoming the tool and carrier of social transition. Without doubt, "community" in the sense of Toennies has no means to explain modern cities, but this historical defect has not made it become a "dead concept, and the implication of "communication", "sharing" and "common sense" that "community" has makes it impossible for it to withdraw from people's thinking and value selection.

There are three viewpoints in the quantitative stipulation of urban community, namely, theory of street district, theory of residents committee district and theory of district between residents committee district and street district. The theory of street district runs counter to the reform value orientation of "transfer from street residence system to community system". Street office is the field agency of the government, with the characteristics of "being only" and the motive of pursuing political performance, so work of street office has strong administrative color. The legal functions of street office do not match with burdening of community affairs, which may also result in misplacement of functions of street office. However, although the theory of district between residences committee district and street district emphasizes regulation of the scale of community, it lacks manipuility in practice. On one hand, readjustment of the administrative division that has already been formed will necessarily 
cost enormous adjustment expense. On the other hand, there lacks a definite direction in the organizational carrier who should assume community affairs. If both the street district and residents committee are undertakers, this will obviously lead to conflicts in sphere of jurisdiction and contradiction will also appear between the two organizations with totally different features. If neither of these two organizations is the undertaker and a third organization is the undertaker, then at least, such an organization can not be reconstructed for the time being, and even if it can be reconstructed, it is uneconomical. Comparatively speaking, it is more realistic and feasible to position community from the perspective of residents committee since it can combine organically the subject of the idea of community (residents) and the subject of action (residents committee).

Whether at the psychological level or at the regional level, definition of urban community has a strong purpose. In 1919, the famous American community worker Mary Follett put forward the proposition that "community is a process" for the first time, and she emphasized building community based on common interests. Importance of the proposition of "process" is that, it acknowledges that the real community is an ideal and is open for construction. Therefore, comprehension of urban community should emphasize what it ought to be, but not what it is or what it has already been. As such, urban community can be essentially defined as a harmonious community with psychological and regional congruence. Urban community is not merely community in its psychological sense, and in the same time, is community in its regional sense. Psychology and region are important constituent factors of urban community, neither of the two dispensable. "Congruence" means unification of urban community at the psychological level and at the regional level. "Congruence" is different from "doublication", because unification under "doublication" is mechanical and closed, while unification under congruence is organic and open. Harmonious community is the target of urban community development and the theme of construction. In terms of target orientation, harmonious community is specific reflection of constructing a harmonious society and embodies characteristics of the contemporary times; in terms of practical operation, "the theory of rebuilding" should be brought into construction to actively promote community rebuilding and to construct a harmonious community; in terms of rebuilding, this does not only include "creation of things" at the regional level, but also includes "creation of human being" at the psychological level. Rebuilding of a harmonious urban community is actually a process of transfer of urban community management mode from "heteronomy" to "autonomy".

\section{Management model: heteronomy and autonomy}

From the perspective of management essence and association of model attributes, management models of urban community can be classified into heteronomy and autonomy. Although some transitional features are manifested in transfer from heteronomy to autonomy, it is not enough to cover the overall attributes of the models. "Dichotomy" is helpful to recognize disadvantages of heteronomy and to get a mastery of the essence of autonomy, so as to strengthen motive of transfer of the models.

There are three typical patterns in heteronomy, namely, unit control under the unit system, street control under the street residence system and company control under the tenement system. To dialectically look upon the model of heteronomy, without doubt, it plays a positive role under particular conditions, but is also has some insuperable disadvantages. "Unit community", "street community" and "tenement community" under control of heter-organization is incomplete and malformed, and it is relatively appropriate to describe it with "sub-community": its internal value is seriously underestimated, its community role is not clear, its community functions shrink, its community mechanism is runtish and its residents participation degree is relatively low. Generally speaking, it is uneconomical and unsustainable to evaluate heteronomy from the perspective of development and variation. By contrast, autonomy is a kind of governance mechanism with low cost and high profits. To compare heteronomy and autonomy with free love and arranged marriage, the former being "autonomy" and the latter being "heteronomy". Free love is superior to arranged marriage, and the reason for an arranged marriage to be harmonious in the end is that, it goes through a conversion process, from formerly arrangement by parents to later free love.

The model of autonomy refers to self-governance of self-organization of the community on community affairs, mainly including the following four aspects: one is self-organization, the organizational carrier of autonomy of community. Self-organization exists within the community and is not constituted by enforcement of specific external commands, and is organism of "self-organizing, self-creating and self-evolving". Residents committee is the self-organization that has more legal status and mass foundation and the subject power of self-organization in the community and occupies a core position. Intermediary organization in the community is an important carrier for community to go towards autonomy. Introduction of the concept of "self-organization" breaks through the narrow thinking that "autonomy of the community equals to autonomy of residents committee." Secondly, affairs of community are limited. Some believe that, all affairs in the community that are related with residents 
belong to affairs of the community, which, as a matter of fact, this implies the concept of "unlimited community", which will necessarily lead to chaos of the agent of community management and will cause weakening of the attribute of community autonomy. The three parties of community, market and government have their own functional boundaries, affairs of community has a paralleling relation with administrative affairs and market affairs, but not relation of subordination. Thirdly, self-governance is manifested in "four selves" and "four democracies". "Four selves" are an important symbol to judge maturity of autonomy of the community and "four democracies" are the institutional conditions required by "four selves". The logic line of self-governance is from "the four democracies" to "the four selves", that is, members of the community settle differences, enhance trust, come to common view and gradually form the behavior model of "four selves" by identifying and practicing "the four democracies", and finally enter the state of "self-governance". Fourthly, right of autonomy is combination of power transferred of heter-organization and endogenous power of self-organization. The process from heteronomy to autonomy is one in which rights and resources of the community are re-distributed. In practice, people are limited to emphasize the so-called "power alienation", not realizing that right of autonomy is also a systematic process of dynamic growth and self-evolution. Actually, right of autonomy of the community does not only origin from entrustment of heter-organization, but more comes from endogeny of self-organization. Endogenous power and power transferred are "interdependent" in that, cultivation of endogenous power provides broad "space" for transferred power and makes heter-organization "more willing" to transfer its power to self-organization; increase of transferred power offers solid "soil" for endogenous power and makes self-organization more "competent" to take over power transferred from heter-organization.

In fact, autonomy does not mean "confrontation" or "collegiality" behavior. The academic circle has two tendencies for autonomy of the community. First is "autonomy outside the government", which regards relation between government and community as antagonistic relation and negates role of the government in autonomy of the community. This viewpoint emphasizes separation of powers between the government and the community to ensure that community organization becomes an independent authority agent, which obviously has its rational aspect. However, it over-emphasizes "right autonomy" of the community and might go towards the extreme of absolute separation between the government and the community, and furthermore, leads to "anarchy" and even "national crisis". As a matter of fact, even in US, a country with higher degree of autonomy, the relation between the government and the community is merely a relative separation, and autonomy of the community is unlikely to be realized without direction and support of the government. Second is "autonomy of the government and the community", which regards relation between the government and the community as a cooperative relation and emphasizes intervention of the government in autonomy of the community. In a long run, without doubt, "cooperation" and "autonomy" have their positive significance, but a "fatal" pre-condition is neglected, that is, only "separation of government administration from commune" can make it possible to really realize cooperation "without classification of classes". At the initial stage, implementation of "collegiality" is extremely likely to be trapped in the tight corner of "heter-organization". The government has a strong "capture power" against the community owing to its resource superiority, so the cooperative relation usually evolves into intervention of the government to the community, while the degree of administrative power makes an inverse proportion to the degree of autonomy of a residential district.

\section{Condition of transfer: regulation and guarantee}

It is the primary condition for transfer of the management mode of urban community to establish autonomy-oriented "community system". Firstly, to straighten out organizational relation and to confirm the core status of residents committee. The key to straighten out relationship between street district and residents committee is to define their own functions in a scientific way, to make clear the intervention behavior and direction behavior of street district, to carry out "system of refusal" and "system of charging", to get rid of the relationship of administrative subordination between superiors and inferiors and to recover the equal relationship of leadership and assistance. We can adjust relation between the residents committee, tenement company and proprietor committee as below: in the short run, proprietor committee can be categorized as a subordinate body of residents committee, under direction and supervision of residents committee, while proprietor committee can establish a relationship of entrustment and fiduciary with tenement company; in the long run, the proprietor committee can be gradually cancelled. Then, functions that are originally assumed by proprietor committee can be transferred to residents committee and residents committee can directly establish a relationship of entrustment and fiduciary with tenement company. The residents committee district in the future should include several tenement administration areas. Residents committee can have a direct conversation with several tenement companies, so as to change the state of "one-to-one" between proprietor committee and tenement company in the past and embody the principle of "simplification and high efficiency". Secondly, to optimize operational 
mechanism and build functional community organizational network. According to the principle of "separation of legislation and execution", the operational structure of the community can be divided into the leadership level, decision making level, discussion making level and executive level. As a leadership level, the Party organization has the function to grasp the direction of community autonomy in politics and ideology. Residents conference is the decision making level, which represents the fundamental interests of all residents and is the organ of highest authority in the community. Consultation conference is the discussion making level and executes the functions of consultation, discussion and democratic supervision during the closing period of resident conference. Residents committee is the executive level of the community, which is directly voted by residents conference, accepts its supervision and reports to residents conference, and manages community affairs according to the principle of "self-management, self-education, self-service and self-supervision".

Fourthly, to straighten out relationship between setting of the community of setting of residents committee. It is the basic guarantee for transfer of management mode of urban community to carry out autonomy-oriented "community law". The government should formulate "Autonomy Law of Urban Community", which ought to carry out "the idea of autonomy" in a comprehensive way, define connotation of community autonomy, community self-organization and community affairs, embody the operational structure and interactive relations between the four levels of leadership, decision making, discussion making and executive and provide legal foundation for promoting autonomy of urban community. The government should revise "Organization Law of Urban Residents Committee" as below: firstly, to strengthen relationship between urban community and residents committee and correct its title into "Organization Law of Urban Community Residents Committee"; to add the content of "self-supervision" in the second Article, which is more corresponding with demand of autonomy and reflects the spirit of the times and to add the content of "being responsible for performing the function of autonomy and management of the community" so as to establish status of residents committee in autonomy management of the community. Secondly, on the basis of ensuring coherence of legal terms and compatibility of content, to try to avoid unnecessary repetitions, and the Eighteenth Article can be deleted and put in "Autonomy Law of Urban Community". Thirdly, to subdivide functions of residents committee and Article Five can be subdivided into autonomy function, assistance function, direction function and supervision duty. Fourthly, to straighten out relationship between setting of the community and setting of the residents committee. The Eighth Article should be modified as "community is set up by the people's government in cities and municipal districts not divided into districts according to conditions of residents' inhabitancy and based on the principle of being convenient for self-governance of residents, being convenient for management of service and being convenient for integration of resources. Any revocation and adjustment of the scale of the community is decided by the people's government in cities and municipal districts not divided into districts." Fifthly, to eliminate inequality between local government and the residents committee in terms of economy. The Twenty-sixth Article should be modified as "working funds of the residents committee should be listed into the financial budget according to certain proportion of the total local revenue and should be provided directly by the country to residents committee. The scope and standard of wages of members in the residents committee should be stipulated by the people's government in cities and municipal districts not divided into districts or people's government of higher levels. After agreement of the conference of residents, wages of members of the residents committee can be supplemented by the economic income of the residents committee."

To develop autonomy-oriented community economy is an important basis for transfer of management mode of urban community. Community autonomy does not only have the particular connotation of "political autonomy", but has extremely high degree of "economic autonomy". Establishment of economic conditions is based, first of all, on establishing independent community financial system. The residents committee should be endowed with qualification of a legal person and a financial management center should be set up to be responsible for financial management of the residents committee. All income and expenses of the residents community have to be brought under unified management of the financial management center. We should strengthen the financial budget of the community, make a balance of payments and reduce financial risks of the community. At the same time, we should carry out the disclosure system of finance, let the residents committee dominate its funds independently, publicize the financial revenue condition of the residents committee and accept supervision of the residents conference and residents. Urban community economy is both distinguished from "public sector economy" and "private sector economy", and should be regarded as a sort of economic pattern within the scope of "the third sector economy". Considering its behavioral agent, urban community economy is an intermediate organization of the residents committee and the community, and should not include other organizations as street district, enterprise and public institution, etc; considering its attribute, urban community economy is a kind of service-based economy, and its sociality, namely, its non-profit public benefit is the most important essential stipulation; considering its development goal, it should correspond with the goal of construction of the 
community, that is, to pursue the maximization of social welfare. We should put forth effort from two aspects to develop community economy. Firstly, to reinforce the leading function of the government and to ensure the continuity of urban economic development. At present, anomie of spatial use and shortage of development space have become the bottleneck of urban economic development. The government should offer positive guidance and provide or open space of development to promote healthy and orderly development of urban economy. According to resource advantages of the community, we should confirm the emphasis and order of industrial development and direct development of community economy into the track of industrial policy to avoid blindness in development. Secondly, to set up "the committee of urban economic management" to ensure coordination urban economic development. Since the organizational carrier of community economy is not an independent economic entity, we need to establish a special institution to take responsibility for development of the community economy and to coordinate, standardize and manage in a unified way the operational behaviors of all economic organization carriers.

It is the core approach for transfer of urban community management mode to improve the self-governance-oriented self-organization competence. Transfer of management mode means exchange of the carrier that assumes affairs of the community, that is, to transfer from "heter-organization" to "self-organization". The capacity of self-organization means the competence of self-organization, as a complex of resources, to effectively perform functions and realize the goal of self-governance. As a particular convergence pattern, the competence of community self-organization is a kind of structural force, and is constituted by such competence as "integrating resources, converging the common sense, resolving conflicts, promoting harmony, self-adaptation and realizing innovation", etc. Endogeneity and autonomy are the essential attributes of the self-organization competence of the community. Once resources of community self-organization are formed, they will be stored as the energy of the organizational system, and when these resources are "transferred" and utilized in different contexts, they will be manifested specifically as all kinds of capacities. The self-organization competence of the community means the explicit form presented after all sorts of resources are organized to get together, allocate and utilize for a specific purpose. It can be seen, resources are the generation source and improvement source of the self-organization competence of the community. "Hard resource" of the community self-organization is constituted by such tangible resources as material resource, financial resource and human resource, etc. Material resource is the basic resource, and financial resource is the central resource and is the economic foundation for the organization to maintain continuity and fulfill its functions, while human resource is the leading resource and is the "essence of autonomy of the community". "Soft resource" plays its role with an intangible power and is constituted by such spiritual resources as authority, system and culture, etc. Formation of authority depends on the benign arrangement of the system and improvement of the competence of participation of the community. It is indicated by experiences, an organization with low institutional degree is not only a weak organization, but also a bad organization. Cultural maintenance is the important manifestation of the self-organization competence of the community. To remould culture of the community needs to start from the following several aspects: improving the cultural system of the community, remoulding and cultivating modern community spirit, setting up the mechanism of co-construction and sharing of community culture and exhibiting culture of the community. It should be pointed out, as a result of the influences of historical traditions and urban modernization, "culture of autonomy" of the community is extremely in shortage and its theoretical study is still "black". However, "culture of autonomy" is the "code" to constitute self-organization behaviors of the community and it vigorously dominates the autonomy behaviors of the subject. Therefore, to remould the culture of the community ought to bring prominence to the core element of "culture of autonomy". In other words, it is a realistic and unavoidable theme of subject to construct the core of "culture of autonomy" and direct the overall moulding of the community culture in a unified way.

\section{Reform means: external driving and endogenesis}

Under the background of system transformation and social transition, the fact that "heteronomy model" of urban community goes towards disintegration represents the general trend and is irreversible. However, from explanation of transfer conditions, it can be found that, the process from "heteronomy" to "autonomy" is not simply a linear transition, but an extremely complicated "two-way interaction" process, which is systematic and difficult and lasts long. There is no doubt that, building of this systematic project has its reform significance. Since there is no existing model to refer to at home and it is appropriate to mechanically copy foreign experiences as a result of different national situations, we have to explore suitable reform means in order to avoid blind advancement and detours.

From the perspective of motive mechanism, means of reform of urban community from heteronomy to autonomy mainly include "external driving" and "endogenesis. External driving reform mainly depends on 
intervention, direction and push of external power and fulfillment of community autonomy is the outcome of external thrust, which follows the route logic of "from the outside to the inside and from top to bottom". Activation of this kind of reform means is usually based on external demand, but not internal appeal and advances with a compulsory means, which has strong passivity for the internal community. The term "endogenesis" mainly originates from biology, which describes an organization or entity that is within an organism and is formed through this organism. Growth of an organ is always the outcome of endogenesis, and people can promote growth of a life. However, in the last analysis, growth of a life means that this life itself is growing. Compared with external driving, endogenesis emphasizes that reform mainly depends on activation and accumulation of internal power of the community and fulfillment of autonomy of the community is the outcome of power of community itself, which follows the route logic of "from the inside to the outside and from bottom to top". Reform of endogenesis starts out from demand and will of residents, centered with establishment of competence of community and advances in a soft, natural and progressive way, which has strong subjectivity to community itself.

Selection of reform means is an important issue encountered in autonomy practice of the community and it directly relates with success or failure of reform. However, over the years, the issue of reform means has not aroused enough attention in the academic circle and is in an extremely marginal position in study on theories of community management. Under direction which is short of theory, it is a common reality that people usually com to emotional cognition about "semantic dependency between endogenesis and autonomy", so they may form such a thinking set: they think that external driving "is something bad by nature", and reform with this method can not realize community autonomy in its real sense; by contrast, endogenesis is the core idea of community autonomy and is the reform means that is most matching and appropriate. Influence of the thinking set will naturally cause people to make a choice "leaning to one side": they exclude and negate external driving means with such a psychology of "the first impressions is firmly entrenched", while they hold endogenesis in high esteem with the mind of "their own wishful thinking". It is proved through practice, excessive worship on the type of endogenesis will not only make the government and the community trapped in an extremely passive state, but can also make it difficult to get an ideal effect of autonomy of the community and may even lead to an outcome that deviates from the autonomy target of the community. On one hand, realization of autonomy per se is essentially a process of game between the government and the community self-organization. Thus, exclusion of the external driving type may make the government more justified in being idle to "contribute their own power". On the other hand, as a result of limited resources and lack of self-organization capacity, self-organization of the community is unable to administer affairs of the community in an effective way. And when a huge leak appears in administration of community affairs, the self-organization has to rely on external power of the government to resolve these affairs, which may lead to "another dependence" on the government. As such, not only the degree of autonomy has not been improved, but that control of heteronomy is aggravated.

Objectively speaking, the two reform means of external driving and endogenesis have no distinction between good and bad. When either of the two means is adopted to reform but fails to achieve the target anticipated, as the subject selected, the first thing to do is not to evaluate advantages and disadvantages of this means of reform, but to reflect on whether this sort of reform means has selected right target and used right stages. "Target" is mainly contrasted with conditions required for "transfer from heteronomy to autonomy", and different conditions and attributes determine different powers for creating conditions, which determines different reform means adopted. Specifically speaking, the system depends on support of the national policy and design of the social system, while the law is formulated by a legislative body and has its authority and enforceability. Without doubt, creating of these two conditions should resort to the external driving means. The core of organizational conditions lies in establishment of self-organization competence of the community and since it mainly relies on the internal power of the community, the endogenesis means should be selected. Channels of the economic sources of the community should include government allocation, "financial aid of the third party" and self-financing of the community, and accumulation of economic conditions should select the mixed pattern of external driving means and endogenesis." "Stage" is mainly contrasted with the development process of "transfer from heteronomy to autonomy", and this process is accompanied by contest between loss and gain of heteronomy and autonomy, which is manifested as obvious feature of stages. The whole process to compare the two powers can be classified into the three periods of heteronomy, mixed governance and autonomy, and difference of the development stages determines difference of the reform means. During the period of heteronomy, it is quite difficult for self-organization of the community to contend against other organizations as a result of its great disparity with other organization. If the endogenesis means is used, this obviously lacks possibility of reality. Thus, only if the means of external driving is used during this period, can it seek for a breakthrough to break down "shackles" of the control community of heter-organization and furthermore, weaken 
heter-organization and broaden the survival space of the community self-organization. During the mixed period, self-organization of the community achieves a balance of power with heter-organization, but competition between the two is still quite fierce, because the self-organization of the community at this time has already obtained a "jetton" to contend with heter-organization and will try to prevent weakening of its own power. In order to further enhance the self-organization competence of the community and avoid it from "turning back the clock", the means of external driving and the mixed means should be used during this period. During the autonomy period, use of endogenesis can guarantee continuity of urban community development. In a word, it is not that a single reform means is used in transfer from "heteronomy" to "autonomy", but that appropriate choice is made by taking into consideration of transferring conditions and development stages.

\section{References}

Chen, Weidong. (2004). Autonomy of Community: Self-organization Network and Institutional Setting. China Social Sciences Press.

Follett,M.P. (1919). Community Is a Process. Philosophical Review, 28, p576.

Gao, Jianguo. (2006). Theoretical Concept and Research Perspective of Community. Learning and Practice, No. 10.

Hu, Zongshan. (2004). Practice of Autonomy in Community. Wuhan Publishing House.

J.G. March \& J. P. Olsen. (1989). Rediscovering Institutions. New York: Free Press, p158-160.

Li, Guoxiang. (2006). Thoughts on Revision of "Organic Law of the Urban Residents Committees of the Peoples Republic of China". Guangxi Social Sciences, No. 1.

Lin, Shangli. (2003). Democracy and Governance in Community: A Case Study. Social Sciences Academic Press.

Shan, Jinging. (2005). Community Emotion and Community Construction. Social Sciences Academic Press.

Tan, Zhilin. (2006). Selection of Routes for Community Building in Taiwan and Community Re-building in Mainland China. Chinese Public Administration, No. 10.

Tang, Yalin \& Chen, Xianshu. (2003). Community Self- rule: Rejuvenation and Development of Democracy at the Basic Level of Urban Society. Academics in China, No. 6.

Wang, Qingshan \& Liu, Jitong. (2004). Study on Models of Community Construction in China. China Social Sciences Press.

Yang, Guihua. (2008). Rebuilding Community Culture to Upgrade the Cultural Maintenance of Community. Journal of Shanghai University (Social Sciences Edition), No. 5.

Zhang, Chao. (2004). Community Economy and Innovation of Its Management Mechanism. Academia Bimestris, No. 2. 